\title{
Current developments in imaging for deep vein thrombosis
}

\author{
Yolanda Lee *, FHKCR, FHKAM (Radiology) \\ Department of Imaging and Interventional Radiology, Prince of Wales Hospital, Shatin, Hong Kong
}

Hong Kong Med J 2015;21:96-7

DOI: $10.12809 / \mathrm{hkmj} 154545$

Venous thromboembolism (VTE), including deep vein thrombosis (DVT) and pulmonary embolism (PE), is believed to be less common in Asian countries than in the West. However, recent studies on the incidence of VTE in Asian population have shown that the frequency is comparable to that of the West. Historically, conventional diagnostic catheter venography was the imaging modality of choice for diagnosis of DVT. With the introduction of ultrasound (US) in the 1980s, it has been widely adopted as the preferred imaging investigation for its non-invasive nature and accuracy.

Most cases of DVT are believed to begin around the leaflets of venous valves in the calves and propagate cranially. A smaller portion begins in the upper thigh, pelvis, or lower abdomen due to obstruction and can propagate caudally. The incidence of DVT in the upper limbs has been on the rise with increasing use of central venous catheters, pacemakers, and automated implanted defibrillators, but the overall incidence remains low. Moreover, the prevalence of $\mathrm{PE}$ in patients with upper extremity (UE) DVT (2\%) is much lower than that of lower extremity (LE) DVT (14.5\%). ${ }^{1}$ A multicentre report of 5451 patients revealed an incidence of unilateral LEDVT in $77 \%$, bilateral LEDVT in $12 \%$, and UEDVT in $11 \% .^{2}$ In a local regional hospital in Hong Kong, US diagnosis of DVT (UEDVT and LEDVT) was seen in 822 (7.6\%) patients, of which UEDVT was seen in $4.1 \%$ and LEDVT in $95.9 \% .^{3}$

Ultrasound is widely recognised as the preferred imaging of choice for suspected proximal LEDVT and UEDVT., ${ }^{4,5}$ It is cost-effective, noninvasive, readily available, portable for critically ill patients, and devoid of ionising radiation. In a recent meta-analysis, US was shown to have a high sensitivity (range, 93.2-95.0\%) and specificity (range, 93.1-94.4\%) for diagnosis of proximal DVT. ${ }^{6}$ On the other hand, US is much less accurate for the diagnosis of calf DVT but the significance of and therapy for isolated calf vein DVT remain controversial. The major sonographic criterion for DVT is failure of complete luminal collapse on probe pressure. Therefore, grey-scale US is routinely supplemented by colour flow and spectral Doppler to avoid false-positive results in areas where probe pressure is ineffective. Colour Doppler US is also useful in showing the degree of venous occlusion (ie partial vs complete occlusion). Spectral Doppler US could serve as an indicator of proximal venous obstruction when monophasic waveform is detected in the common femoral vein. The augmentation method, previously believed to increase sensitivity of diagnosing proximal DVT, has recently been shown to be of limited value in the diagnosis of proximal DVT in a series of almost 2000 examinations. ${ }^{7}$

Potential pitfalls of US include false-positive findings in the adductor canal, in portions of the subclavian vein deep to the clavicle, in severely oedematous limbs, and in patients with large body habitus when probe pressure is ineffective. Colour and spectral Doppler US could help in establishing patency of the vein in these conditions. Other potential pitfalls of US include false-negative results in cases of duplicated femoral vein (when only the patent limb is identified), obscured inferior vena cava and iliac veins (which is always a challenge on routine US), and failure to differentiate between acute-on-chronic DVT and chronic DVT (up to 50\% of patients with DVT have residual abnormality on follow-up US).

Magnetic resonance venography (MRV) is recommended in the American College of Radiology Appropriateness Criteria ${ }^{4}$ to be the imaging investigation of choice for evaluation of pelvic or thigh DVT if US is non-diagnostic and as an initial imaging investigation of choice for suspected central vein thrombosis in the thorax. Magnetic resonance has the advantage of not exposing patients to ionising radiation or iodinated contrast compared with computed tomography (CT). Magnetic resonance venography is also superior to US in evaluating veins above the inguinal ligament and is able to show potential sources of extrinsic venous compression in the pelvis. The main limitation to the use of MRV is its lower cost-effectiveness and limited availability, especially in acute clinical settings. In general, contrast media-enhanced MRV is preferred because of higher reproducibility and lower possibility of image artefacts. When gadolinium is contraindicated, non-contrast MRV remains a useful tool for diagnosing DVT by the use of a variety of pulse sequences and techniques such as spin echo, fast-spin echo, time-of-flight, phase contrast, and 
steady-state free precession, when the limitations of reproducibility and artefacts being understood.

Computed tomography venography (CTV) has been shown to be comparable to US in diagnosing proximal DVT. A recent meta-analysis showed CTV has sensitivity and specificity ranging from $71 \%$ to $100 \%$ and from $93 \%$ to $100 \%$, respectively, for the diagnosis of proximal DVT. ${ }^{8}$ For diagnosis of DVT in the calf veins, CTV may be superior to US. Due to significantly higher radiation dose and the risk of iodinated contrast media, CTV should be reserved for when MRV is not available or is contra-indicated. In selected patients, CTV could be incorporated into CT pulmonary angiography (CTPA) for evaluation of both PE and proximal DVT, but it should not be routinely performed for all patients undergoing CTPA.

Given the invasive nature and risks similar to CT (exposure to ionising radiation and iodinated contrast medium), the use of conventional venography in the diagnosis of DVT is limited to a few specific scenarios: inconclusive non-invasive imaging result, when thrombolysis is planned, prior to placement of inferior vena cava filters, and evaluation of central DVT in the proximal arms and thorax.

In summary, US is the most cost-effective non-invasive imaging method for suspected DVT in proximal LEs and in UEs. In cases of a negative initial US result but with persistent symptoms, follow-up US would be helpful to exclude proximal extension of DVT, if any. In addition, MRV and CTV can be used as alternative imaging methods for patients with a non-diagnostic US, who are unable to undergo US, or who are highly suspected to have pelvic DVT. Conventional venography is reserved for a few specific scenarios in modern day practice, usually as a prelude to thrombolysis.

\section{References}

1. Levy MM, Albuquerque F, Pfeifer JD. Low incidence of pulmonary embolism associated with upper-extremity deep venous thrombosis. Ann Vas Surg 2012;26:964-72.

2. Goldhaber SZ, Tapson VF; DVT FREE Steering Committee. A prospective registry of 5,451 patients with ultrasound-confirmed deep vein thrombosis. Am J Cardiol 2004;93:259-62.

3. Chung AS, Luk WH, Lo AX, Lo CF. Duplex sonography for detection of deep vein thrombosis of upper extremities: a 13-year experience. Hong Kong Med J 2015;21:107-13.

4. Hanley M, Donahue J, Rybicki FJ, et al. American College of Radiology: ACR Appropriateness Criteria ${ }^{\circledR}$. Clinical condition: suspected lower-extremity deep vein thrombosis. Available at: https://acsearch.acr.org/ docs/69416/Narrative. Accessed 28 Feb 2015.

5. Dill KE, Bennett SJ, Hanley M, et al. American College of Radiology: ACR Appropriateness Criteria ${ }^{\circledR}$. Clinical condition: upper extremity swelling. Available at https:// acsearch.acr.org/docs/69417/Narrative. Accessed 28 Feb 2015.

6. Lewis BD, James EM, Welch TJ, Joyce JW, Hallett JW, Weaver AL. Diagnosis of acute deep venous thrombosis of the lower extremities: prospective evaluation of color Doppler flow imaging versus venography. Radiology 1994;192:651-5.

7. Lockhart ME, Sheldon HI, Robbin ML. Augmentation in lower extremity sonography for the detection of deep venous thrombosis. AJR Am J Roentgenol 2005;184:419-22.

8. Thomas SM, Goodacre SW, Sampson FC, van Beek EJ. Diagnostic value of CT for deep vein thrombosis: results of a systematic review and meta-analysis. Clin Radiol 2008;63:299-304. 